\title{
Prognostic Value of Standard Parameters as Predictors for Long-Term Renal Replacement Therapy after Liver Transplantation
}

\author{
Kilian Weigand ${ }^{b}$ Eva Bauer $^{b}$ Jens Encke ${ }^{a, b}$ Jan Schmidt ${ }^{c}$ \\ Wolfgang Stremmel $^{\mathrm{b}}$ Vedat Schwenger $^{\mathrm{d}}$ \\ a Department of Medicine, Johanna-Etienne-Krankenhaus, Neuss, Departments of ${ }^{\mathrm{b}}$ Gastroenterology and \\ Hepatology, 'General Surgery, and ${ }^{\mathrm{d}}$ Nephrology, University Hospital Heidelberg, Heidelberg, Germany
}

\section{Key Words}

Liver · Transplantation • Hemodialysis • Renal insufficiency • Mortality

\begin{abstract}
Chronic kidney disease has become increasingly prevalent after liver transplantation (LTPL) because outcome and survival rates have improved. Chronic kidney insufficiency is most likely associated with increased morbidity and mortality. The challenge is to identify patients who will be in need of long-term renal replacement therapy (RRT) after LTPL. We analyzed 208 liver transplant recipients with respect to mortality, associated laboratory values, underlying liver disease, immunosuppressive protocol and the need for RRT. Longterm RRT was defined by the need for RRT 3 months after LTPL. Altogether, $5.8 \%$ of the surviving study patients remained in need of RRT 3 months after LTPL. All of these patients continued to need RRT throughout the study period (2 years). The need for RRT significantly increased the 2-year mortality rate 4.3 -fold, from 15.4 to $66.7 \%$ ( $p=0.004$ ). Comparison of laboratory and clinical parameters at the time of LTPL revealed no significant differences for creatinine, albumin and MDRD between patients undergoing hemodialysis 3 months after LTPL and patients without RRT. Comparing
\end{abstract}

mean urea, a difference was observed. However, multivariate regression analyses using easy-to-observe demographic or laboratory parameters failed to generate a model to predict the need for RRT after LTPL. In addition, a comparison of underlying liver disease and immunosuppressive regimes identified no significant differences. Taken together, patients who were on hemodialysis 3 months after LTPL were also on hemodialysis 2 years after LTPL or until death. RRT 3 months after LTPL may predict the risk for chronic renal insufficiency and is associated with significantly increased mortality.

Copyright $\odot 2011$ S. Karger AG, Basel

\section{Introduction}

Chronic kidney disease is a severe problem after liver transplantation (LTPL), as it is associated with enhanced morbidity and mortality [1-3]. Therefore, kidney function should preoperatively be evaluated if the patient is at risk of losing adequate kidney function after LTPL [4]. The number of candidates for LTPL with kidney dysfunction has increased since serum creatinine is a component of the MELD score [5]. Liver recipients who are likely to have higher stages of chronic renal disease or who are in

\section{KARGER}

Fax +41613061234 E-Mail karger@karger.ch www.karger.com

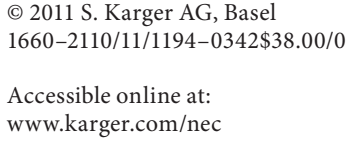

Kilian Weigand, MD

Department of Gastroenterology and Hepatology, Medizinische Klinik IV

Im Neuenheimer Feld 410

DE-69120 Heidelberg (Germany)

Tel.+496221 5638 747, E-Mail kilian_weigand@ med.uni-heidelberg.de 
Table 1. Demographic and clinical characteristics of 208 liver transplant recipients from January 2004 to December 2006 at the University Hospital of Heidelberg, Heidelberg, Germany

\begin{tabular}{lc}
\hline Variables & Value $(\mathrm{n}=208)$ \\
\hline Age of patient, years & \\
Mean (SD) & $47.6(14.3)$ \\
Median & 51 \\
Gender & $137(65.9)$ \\
Male & \\
Indications for transplantation ${ }^{\mathrm{a}}$ & $33(15.9)$ \\
Hepatitis C & $19(9.1)$ \\
Hepatitis B & $61(29.3)$ \\
Alcoholic liver disease & $3(1.4)$ \\
Wilson's disease & $11(5.3)$ \\
Autoimmune hepatitis & $4(1.9)$ \\
Hemochromatosis & $46(22.1)$ \\
Hepatocellular carcinoma & $61(29.3)$ \\
Others & $44(21.2)$ \\
Necessity of hemodialysis & $12(5.8)$ \\
At time of transplantation & \\
3 months after transplantation & $21(10.1)$ \\
Immunosuppressive regime ${ }^{b}$ & $84(40.8)$ \\
Cyclosporine & $49(23.6)$ \\
Cyclosporine and azathioprine & $34(16.3)$ \\
Tacrolimus & $20(9.6)$ \\
Cyclosporine and MMF & \\
Tacrolimus and MMF & \\
&
\end{tabular}

Values are $\mathrm{n}(\%)$, unless otherwise stated. MMF = Mycophenolate mofetil.

a Cumulative incidences; two or more underlying diseases were counted to each of the listed diseases.

${ }^{\mathrm{b}}$ Immunosuppressive regime at the beginning; for changes in the follow-up time due to various reasons see main text. Every regime included additional methylprednisolone in decreasing dosage over time.

long-term need of hemodialysis should therefore be recommended for combined liver and kidney transplantation $[6,7]$. There are several possibilities to identify kidney disease. One way is simply to check laboratory parameters such as creatinine or urea; another is to calculate functions to estimate the glomerular filtration rate, e.g. by the Modification of Diet in Renal Disease (MDRD) [8, 9]. A superior method may be to measure the 24 -hour collection for creatinine clearance [9]. Furthermore, abnormal kidney function can be detected by anatomic evaluation, urinary electrolytes and osmolarity, proteinuria, or by renal biopsy [10].

Many of these parameters are altered in patients with end-stage liver disease due to reasons like liver failure, hepatorenal syndrome, low muscle mass, poor nutrition- al status, edemas, etc. Therefore, the risk of developing a severe degree of chronic kidney disease after LTPL - requiring renal replacement therapy (RRT) - may easily be under- or overestimated.

In this retrospective analysis, we tried to define easily assessable predictors at the time of LTPL that identify patients who will need long-term RRT.

\section{Patients and Methods}

\section{Patient Cohort}

Patients undergoing LTPL at the University Hospital of Heidelberg from January 2004 to December 2006 were included in this retrospective analysis. Exclusion criteria were: (1) patients who died within the first 4 weeks after LTPL $(n=29)$ with missing follow-up data and different causes of death not necessarily associated with acute kidney insufficiency, (2) patients who were retransplanted within 2 weeks after the first LTPL ( $n=13$; of these patients the second transplant period was included; there was no patient in need of a third LTPL within 1 year after the second transplantation), and (3) patients in need of RRT 3 months prior to LTPL $(n=3)$. All patients with acute renal failure were included in this study. From the 253 screened patients, 208 met the inclusion criteria. Demographic characteristics as well as indications for LTPL, immunosuppressive regimen and the need for hemodialysis are presented in table 1.

\section{Data Collection}

The medical records of the patients included in our study were reviewed for 2-year survival rates, demographic data, underlying liver disease that led to LTPL and the immunosuppressive regimen. Values for creatinine, urea, albumin and MDRD, as well as the need for RRT were recorded. For patients undergoing RRT, the laboratory values before the first hemodialysis were used for calculation. Patients receiving RRT 3 months prior to LTPL were not included due to chronic kidney disease. In addition, MDRD was calculated in two different but commonly used ways [11].

\section{Immunosuppressive Regimen}

The immunosuppressive regimen was based on methylprednisolone, as recommended in the guidelines of the transplant center. Starting with up to $40 \mathrm{mg}$ daily, methylprednisolone was tapered down to $2.5 \mathrm{mg}$ or none after 6-12 months. Maintenance immunosuppression consisted of cyclosporine or tacrolimus. Of the study cohort, 139 patients were started on cyclosporine and 69 patients on tacrolimus. At the beginning of the study period, azathioprine was often combined with cyclosporine for 6 months. Later, mycophenolate mofetil became a frequently used medication in addition to cyclosporine or tacrolimus (table 1).

\section{Statistical Analysis}

Descriptive statistics were used to characterize demographic data and clinical variables. The $\chi^{2}$ test was used to assess the relationship between the mortality of the different groups, the underlying liver disease and the need for RRT, as well as the immunosuppressive regimen and the need for RRT. Mortality was further compared using the Kaplan-Meier curve calculation. For univar- 


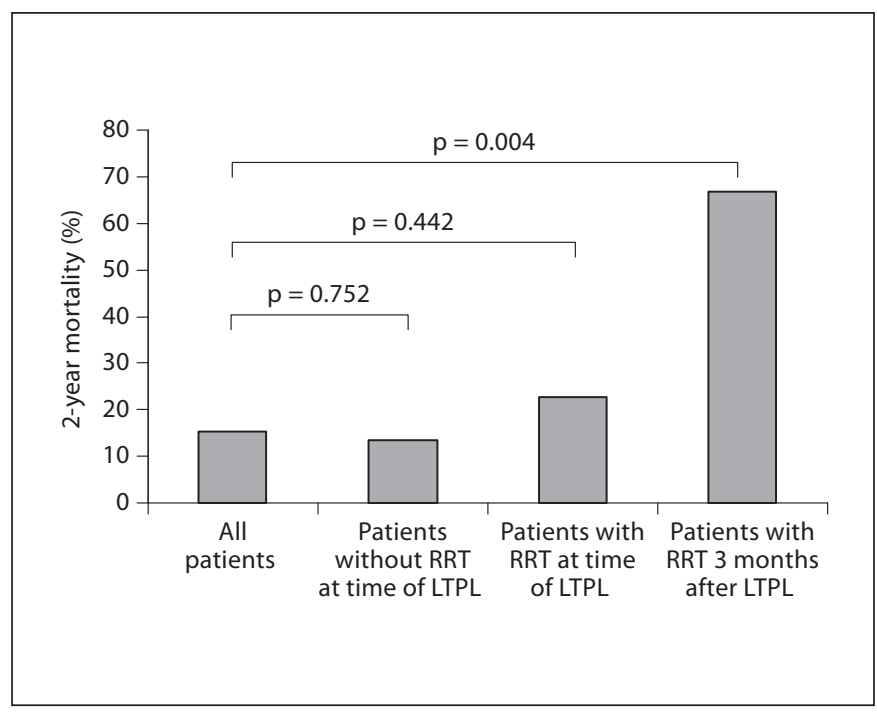

Fig. 1. Mortality of patients in need of RRT within the first 2 years after LTPL.

iate analysis of creatinine, urea, albumin and MDRD, the MannWhitney rank-sum test was used. Multivariate analyses were performed with linear or nonlinear regression models (ANOVA). All statistical analyses were two-sided, and $\mathrm{p}<0.05$ was considered to represent statistical significance. All statistical analyses were performed using the SPSS 17.0 program (SPSS Inc., Chicago, Ill., USA).

\section{Results}

\section{Study Population}

Acute renal failure necessitating RRT was observed in 44 patients (21.2\%) within 3 months prior to or immediately after transplantation. None of these patients was in need of RRT at any point 3 months prior to LTPL as defined in the exclusion criteria. Three months after LTPL, 12 patients $(5.8 \%)$ were still on hemodialysis. All of them were still on RRT 2 years after LTPL (or until their death). Therefore, these patients were considered to suffer from end-stage renal disease. Only 8 of these 12 patients had RRT at the time of LTPL, while 4 developed renal dysfunction after LTPL.

In our study population, therefore, patients with acute renal failure prior to LTPL showed the risk of remaining on RRT with a sensitivity of $67 \%$ and a specificity of $84 \%$. Calculating the predictive values, we found a positive predictive value of $52.7 \%$ and a negative predictive value of $90.5 \%$.

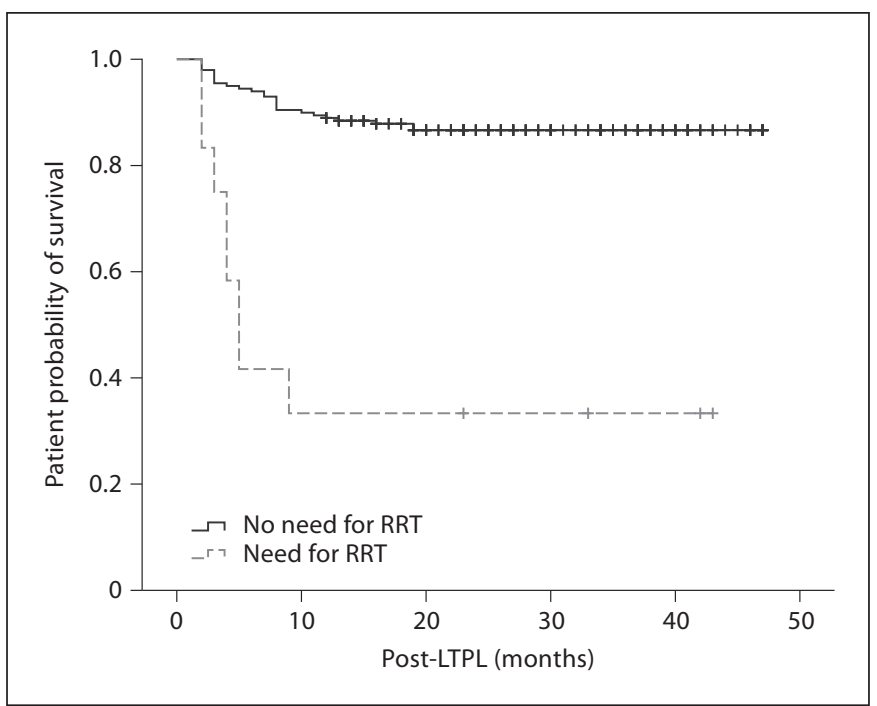

Fig. 2. Kaplan-Meier survival curves of patients with and without the need for RRT 3 months after LTPL.

\section{Need for RRT 3 Months after LTPL Significantly Increased Mortality}

We used a follow-up time of 2 years after LTPL to assess the incidence of mortality in our study population. While we found no association between mortality and RRT at the start of or 3 months prior to LTPL, the 2-year mortality rate significantly increased $(\mathrm{p}=0.004)$ for patients in need of RRT 3 months after LTPL (fig. 1). Figure 2 demonstrates the Kaplan-Meier analysis of patients with or without the need for RRT 3 months after LTPL. Altogether, two thirds of the patients undergoing RRT 3 months after LTPL did not survive the first 2 years after transplantation. The cause of hemodialysis in these 12 patients was acute renal insufficiency of unknown origin in 5 patients, hepatorenal syndrome in 5 patients, cystic kidney disease in 1 patient and diabetic nephropathy in 1 patient.

Regarding graft survival after LTPL, we found no significant correlation with the need for RRT 3 months after LTPL.

\section{Underlying Liver Disease or Immunosuppressive Regimen Did Not Correlate with the Need for RRT after LTPL}

Comparing the underlying liver diseases that led to LTPL with the need for RRT 3 months (and thereafter) after LTPL, we found no significant differences; $p$ values ranged between 0.408 and 0.975 , and are shown in table 2 . 
Table 2. Correlation of patients without RRT 3 months after LTPL and patients with end-stage renal disease (RRT 3 months after LTPL) in respect to underlying liver disease, immunosuppressive regimen and laboratory values (univariate analysis) at time of LTPL

\begin{tabular}{lll}
\hline Patients without & Patients with p \\
RRT 3 months & RRT 3 months \\
after LTPL & after LTPL \\
$(\mathrm{n}=196)$ & $(\mathrm{n}=12)$ \\
\hline
\end{tabular}

\section{Underlying liver disease, $n$}

Hepatitis C

Alcoholic liver disease $\quad 57$

Wilson's disease 3

Autoimmune hepatitis $\quad 11$

Hemochromatosis 4

Hepatocellular carcinoma

Other

$30 \quad 3$

0

4

0

0

44

58

Immunosuppressive regimen, $n$

CsA, U 18

CsA, U, MMF 34

CsA, U, Aza 78

Tac, U 46

Tac, U, MMF 20

Laboratory values (univariate analysis), mean $\pm S D$
Creatinine, $\mathrm{mg} / \mathrm{dl}$

Urea, mg/dl

Albumin, g/l

MDRD, $\mathrm{ml} / \mathrm{min}$

MDRD L, $\mathrm{ml} / \mathrm{min}$

$\begin{array}{ccc}1.05 \pm 0.55 & 1.45 \pm 0.94 & 0.069 \\ 48 \pm 30 & 68 \pm 34 & 0.035 \\ 29.0 \pm 7.5 & 29.3 \pm 7.4 & 0.982 \\ 98 \pm 32 & 77 \pm 37 & 0.066 \\ 90 \pm 80 & 56 \pm 38 & 0.063\end{array}$

CsA = Cyclosporine; $\mathrm{U}=$ methylprednisolone; $\mathrm{MMF}=$ mycophenolate mofetil; Aza $=$ azathioprine; Tac $=$ tacrolimus.
When we correlated the immunosuppressive regimen after LTPL with the long-term need for RRT after LTPL, we again found no significant differences (table 2). No patient with a mycophenolate mofetil-containing regimen was in need of long-term RRT; however, due to the low number of patients, no conclusion is possible.

\section{Multivariate Analysis Revealed that Standard}

Parameters Fail to Predict the Risk of RRT after LTPL

When comparing renal-dependent laboratory values at the time of LTPL of the patients suffering from endstage renal disease after LTPL with those patients who did not undergo hemodialysis 3 months after LTPL, we found no significant differences for creatinine, urea, MDRD and MDRD L (table 2). Urea was the only parameter
Table 3. Multivariate ANOVA linear regression analysis for the risk of RRT 3 months after LTPL

\begin{tabular}{lllll}
\hline Variables & $\mathrm{R}$ & $\mathrm{r}$ value & $\mathrm{R}^{2}$ & $\mathrm{p}$ \\
\hline Age & 0.119 & 0.231 & 0.014 & 0.084 \\
$\quad$ Urea & 0.173 & 0.230 & 0.030 & 0.043 \\
Creatinine & 0.184 & 0.230 & 0.034 & 0.068 \\
MDRD L & 0.188 & 0.230 & 0.036 & 0.112 \\
Albumin & 0.191 & 0.231 & 0.036 & 0.176 \\
Urea & 0.148 & 0.230 & 0.022 & 0.032 \\
Creatinine & 0.162 & 0.230 & 0.026 & 0.064 \\
MDRD L & 0.162 & 0.231 & 0.026 & 0.139 \\
Albumin & 0.166 & 0.231 & 0.027 & 0.218 \\
Creatinine & 0.141 & 0.230 & 0.020 & 0.041 \\
MDRD L & 0.141 & 0.231 & 0.020 & 0.122 \\
Albumin & 0.146 & 0.231 & 0.021 & 0.216 \\
MDRD L & 0.087 & 0.232 & 0.007 & 0.211 \\
Albumin & 0.091 & 0.232 & 0.008 & 0.423 \\
\hline
\end{tabular}

Necessity of RRT is the dependent variable for the analysis with the listed factors cumulatively used as predictor variables. An $R^{2}$ value above 0.4 indicates a significant correlation between the parameters and the necessity of RRT. $p<0.001$ is considered significant. $\mathrm{R}=$ Calculated $\mathrm{ANOVA}$ value; $\mathrm{r}$ value $=$ standard deviation by estimator.

found to reach statistical significance $(\mathrm{p}=0.035)$. Analyzing the parameters age, gender, urea, creatinine, albumin, MDRD and MDRD L in all possible constellations using linear and nonlinear regression analyses, as well as metric or ordinal classifications where appropriate, we found no model with an $\mathrm{R}^{2}$ value above the level of 0.4 (table 3 ). Regarding urea (the only significant parameter in the univariate analysis), matrix cluster analysis showed a complete overlapping of the values of the two groups (data not shown). These data reveal that urea and/or the abovementioned parameters are insufficient to predict the need for RRT 3 months after LTPL in our study population.

\section{Discussion}

We evaluated the long-term consequences of acute renal failure prior to LTPL. In our study population, 5.8\% of the patients remained in need of RRT 3 months after LTPL and all these patients were still on RRT 2 years after LTPL or until their death. Therefore, the need for RRT 3 months after LTPL correlates well to end-stage renal disease. Besides the reduced quality of life for the patient with regular hemodialysis, it is known that acute renal 
insufficiency is associated with increased mortality $[1,2]$. In our study collective, two thirds of these patients died within 2 years after LTPL, which adds up to a greatly increased mortality rate (4.3-fold). Therefore, it would be of great interest to predict the long-term renal impairment after LTPL with easily assessable parameters to schedule such patients for combined liver and kidney transplantation.

There are factors that have been shown to be predictors for the need of RRT after LTPL, such as the presence of kidney dysfunction prior to surgery and a higher serum creatinine concentration $[12,13]$. One retrospective analysis of 724 liver transplant recipients demonstrated a serum creatinine higher than $1.9 \mathrm{mg} / \mathrm{dl}$, blood urea levels above $27 \mathrm{mg} / \mathrm{dl}$, a MELD score greater than 21 and an intensive care stay as important predictors [14]. In addition, calcineurin inhibitors can cause chronic kidney disease months to years after LTPL. Within the early phase after LTPL, only a gradual decline in renal function due to those drugs has been described $[15,16]$. Several models have been described with the intention to predict the need for RRT after LTPL [3, 14, 17]. However, in our study population we found no easily obtainable model to calculate the risk of long-term RRT after LTPL. In particular, we showed that even significant differences in single parameters may not be sufficient to predict the real risk for RRT due to their broad distribution range in the patients on the waiting list for LTPL.

Even though MDRD is not validated for acute kidney disease, it is commonly used in these patients. Therefore, another interesting point we found in our population was the big difference between the calculated initial (before first RRT) MDRD and MDRD L values in many patients. In the subgroup of patients undergoing RRT at the time of LTPL, this difference even reached an almost significant level ( $\mathrm{p}=0.069)$. This leads to the assumption that the two models cannot be used equally for patients awaiting LTPL and may not be a good evaluation of this population at all.

Uncertainty concerning our analysis may be due to the different reasons for starting hemodialysis. Reasons for RRT may be anuria, electrolyte disorders, elevated urea, therapy refractory hydropic decompensation including volume overload, and renal failure following a severe infection. In this study, the reason for RRT was not taken into account. A further differentiation regarding this point would have led to numbers too low for significant regression calculations. Another weakness of our study was that cofactors such as diabetes, hyperlipidemia, blood pressure and the intake of nephrotoxic agents (e.g. NSAR or calcium antagonists) were not assessed in our population. These cofactors influence the renal function and, therefore, most likely influence the need for long-term RRT. However, we do not know which influence these factors have in the short-term course of 3 months - the time period we reviewed. For example, we found no correlation between the intake of tacrolimus or cyclosporine with the need for RRT 3 months after LTPL, despite the knowledge that long-term intake of these drugs results in worsened kidney function $[15,16]$.

The time period for our study was chosen to only include patients before the MELD score became relevant for allocation. Due to this change in allocation, the LTPL collective changed. A comparison of the different collectives would have been biased by many other factors.

This study demonstrates that chronic kidney failure after LTPL greatly increases mortality. The need for RRT 3 months after LTPL may be a good predictor for chronic renal insufficiency. However, it cannot be predicted by regular parameters about which patients remain on hemodialysis after LTPL. The optimal approach to estimate renal function in patients on the waiting list for LTPL remains unclear. Additional diagnostic, e.g. regular kidney biopsy, should be performed to predict which patients would profit from combined liver and kidney transplantation.

\section{Disclosure Statement}

The authors have nothing to disclose regarding this study and declare that they have no competing interests.

References

- 1 O'Riordan A, Wong V, McCormick PA, Hegarty JE, Watson AJ: Chronic kidney disease post-liver transplantation. Nephrol Dial Transplant 2006;21:2630.

-2 Ojo AO, Held PJ, Port FK, et al: Chronic renal failure after transplantation of a nonrenal organ. N Engl J Med 2003;349:931.

-3 Nair S, Verma S, Thuluvath PJ: Pretransplant renal function predicts survival in patients undergoing orthotopic liver transplantation. Hepatology 2002;35:1179.

4 Bloom RD, Reese PP: Chronic kidney disease after nonrenal solid-organ transplantation. J Am Soc Nephrol 2007;18:3031

5 Gonwa TA, McBride MA, Anderson K, Mai ML, Wadei H, Ahsan N: Continued influence of preoperative renal function on outcome of orthotopic liver transplant (OLTX) in the US: where will MELD lead us? Am J Transplant 2006;6:2651. 
6 Davis CL, Feng S, Sung R, et al: Simultaneous liver-kidney transplantation: evaluation to decision making. Am J Transplant 2007;7: 1702 .

7 Brown RS Jr, Lombardero M, Lake JR: Outcome of patients with renal insufficiency undergoing liver or liver-kidney transplantation. Transplantation 1996;62:1788.

8 Gonwa TA, Jennings L, Mai ML, Stark PC, Levey AS, Klintmalm GB: Estimation of glomerular filtration rates before and after orthotopic liver transplantation: evaluation of current equations. Liver Transpl 2004;10: 301.

9 Skluzacek PA, Szewc RG, Nolan CR 3rd, Riley DJ, Lee S, Pergola PE: Prediction of GFR in liver transplant candidates. Am J Kidney Dis 2003;42:1169.
10 Davis CL, Gonwa TA, Wilkinson AH: Pathophysiology of renal disease associated with liver disorders: implications for liver transplantation. Part I. Liver Transpl 2002;8:91.

11 Levey AS, Bosch JP, Lewis JB, Greene T, Rogers N, Roth D: A more accurate method to estimate glomerular filtration rate from serum creatinine: a new prediction equation. Modification of Diet in Renal Disease Study Group. Ann Intern Med 1999;130:461.

12 Contreras G, Garces G, Quartin AA, et al: An epidemiologic study of early renal replacement therapy after orthotopic liver transplantation. J Am Soc Nephrol 2002; 13: 228.

13 Bilbao I, Charco R, Balsells J, et al: Risk factors for acute renal failure requiring dialysis after liver transplantation. Clin Transplant 1998; $12: 123$.
14 Sanchez EQ, Gonwa TA, Levy MF, et al: Preoperative and perioperative predictors of the need for renal replacement therapy after orthotopic liver transplantation. Transplantation 2004;78:1048.

15 A comparison of tacrolimus (FK 506) and cyclosporine for immunosuppression in liver transplantation: the U.S. Multicenter FK506 Liver Study Group. N Engl J Med 1994;331: 1110 .

16 Jain A, Reyes J, Kashyap R, et al: Long-term survival after liver transplantation in 4,000 consecutive patients at a single center. Ann Surg 2000;232:490.

-17 Markmann JF, Markmann JW, Markmann DA, et al: Preoperative factors associated with outcome and their impact on resource use in 1148 consecutive primary liver transplants. Transplantation 2001;72:1113. 